\title{
Metrics of arterial hyperoxia and associated outcome in critical care
}

\author{
HJF Helmerhorst ${ }^{1 *}$, DL Arts², E de Jonge ${ }^{1}$ DJ van Westerloo ${ }^{1}$ \\ From ESICM LIVES 2015 \\ Berlin, Germany. 3-7 October 2015
}

\section{Introduction}

In recent years, emerging evidence has shown the potential risks of arterial hyperoxia, but observational studies failed to indisputably demonstrate its impact on clinical outcome of critically ill patients. Importantly, the lack of a clinical definition of hyperoxia and methodological limitations hamper the interpretation and clinical relevance of these studies.

\section{Objectives}

We aimed to systematically evaluate previously used metrics for defining hyperoxia and associations with outcome of intensive care unit (ICU) admission.

\section{Methods}

Arterial blood gas (ABG) analyses between July 2011 and July 2014 were extracted from the patient data management system (PDMS) database of three large tertiary care ICUs in the Netherlands. Data from all admissions where more than one ABG was available were supplemented with anonymous demographic and admission and discharge data from the Dutch National Intensive Care Evaluation (NICE) registry. Identified oxygen metrics from a systematic review of the literature included the first, highest, worst, and mean arterial oxygen tension $\left(\mathrm{PaO}_{2}\right)$. Worst $\mathrm{PaO}_{2}$ was defined as the $\mathrm{PaO}_{2}$ associated with the lowest $\mathrm{PaO}_{2} / \mathrm{FiO}_{2}$ ratio. In logistic regression models, we analysed the associations between hospital mortality and severe hyperoxia $\left(\mathrm{PaO}_{2}>200 \mathrm{mmHg}\right)$ defined by either identified metric, calculated over the total ICU admission.

\section{Results}

Overall, 14,464 patients were included of whom 1,991 (13.7\%) died before hospital discharge. All identified metrics showed statistically significant associations between severe hyperoxia and hospital mortality. The risk estimates differed substantially between the metrics used for defining hyperoxia $(\mathrm{P}<0.001)$. The strongest effects were found for the mean and the highest $\mathrm{PaO}_{2}$ : crude odds ratio 3.20 [95\% CI 2.21-4.58] and 2.81 [95\% CI 2.49-3.18], respectively. The effect was weaker for the first and worst $\mathrm{PaO}_{2}$ : crude odds ratio 1.22 [95\% CI $1.05-1.41$ ] and 1.17 [95\% CI 1.00-1.36], respectively.

\section{Conclusions}

In this multicenter cohort study, severe arterial hyperoxia was associated with hospital mortality and this association was independent of the used metric for hyperoxia. However, the metric choice greatly influences the effect size for hospital outcome.

\section{Grant Acknowledgment}

This work was financially supported by an unrestricted grant issued by the Netherlands Organization for Health Research and Development (ZonMw).

ADDIN EN.REFLIST

\section{Authors' details}

${ }^{1}$ Leiden University Medical Center, Department of Intensive Care Medicine, Leiden, Netherlands. ${ }^{2}$ Academic Medical Center, Department of Medical Informatics, Amsterdam, Netherlands.

Published: 1 October 2015

doi:10.1186/2197-425X-3-S1-A661

Cite this article as: Helmerhorst et al:: Metrics of arterial hyperoxia and associated outcome in critical care. Intensive Care Medicine Experimental 2015 3(Suppl 1):A661. 